This item was submitted to Loughborough's Research Repository by the author.

Items in Figshare are protected by copyright, with all rights reserved, unless otherwise indicated.

\title{
Numerical study on transition of hydrogen/air flame triggered by auto-ignition under effect of pressure wave in an enclosed space
}

\section{PLEASE CITE THE PUBLISHED VERSION}

http://dx.doi.org/10.1016/j.ijhydene.2017.05.085

\section{PUBLISHER}

Elsevier (C) Hydrogen Energy Publications

VERSION

AM (Accepted Manuscript)

\section{PUBLISHER STATEMENT}

This work is made available according to the conditions of the Creative Commons Attribution-NonCommercialNoDerivatives 4.0 International (CC BY-NC-ND 4.0) licence. Full details of this licence are available at: https://creativecommons.org/licenses/by-nc-nd/4.0/

\section{LICENCE}

CC BY-NC-ND 4.0

\section{REPOSITORY RECORD}

Wei, Haiqiao, Yibao Shang, Jilei Cai, Mingzhang Pan, Gequn Shu, and Rui Chen. 2017. "Numerical Study on Transition of Hydrogen/air Flame Triggered by Auto-ignition Under Effect of Pressure Wave in an Enclosed Space". figshare. https://hdl.handle.net/2134/26095. 


\title{
Numerical study on transition of hydrogen/air flame triggered by auto-ignition under effect of pressure wave in an enclosed space
}

Haiqiao Wei*, Yibao Shang, Jilei Cai, Mingzhang Pan, Gequn Shu, Rui Chen

State Key Laboratory of Engines, Tianjin University, Tianjin 300072, China

Accepted for publication by International Journal of Hydrogen Energy

\begin{abstract}
End gas auto-ignition and transition of flame front are considered as the main causes of severe pressure oscillation in spark-ignition engines, which is one of the major features of knock and superknock. The knowledge of characteristics of auto-ignition, flame front development, propagation of pressure wave and relations between them, still needs to be maintained. In this study, flame front transition induced by pressure wave and auto-ignition are investigated using one-dimensional simulation with detailed chemistry in an enclosed space Calculation cases with different initial thermodynamic conditions are investigated. Mass fraction of $\mathrm{OH}$ is employed as indicator of autoignition progress under variable conditions caused by pressure wave. Different propagation modes of flame front, including subsonic deflagration, detonation and supersonic deflagration, are developed under the effects of both pressure wave and auto-ignition. Results show that mass fraction of $\mathrm{OH}$ could successfully reflect auto-ignition progress, thus indicating occurrence and sequence of auto-ignition at different locations. Transitions from deflagration to detonation and detonation to supersonic deflagration are found to be triggered by sequential auto-ignition with different gradient of auto-ignition progress ahead of flame front induced by pressure wave.
\end{abstract}

"Correspondence author: whq@tju.edu.cn 


\section{Introduction}

As transportation is worsening energy shortage, engine downsizing has become an efficient way of reducing fuel consumption [1]. Compared with normal engine, smaller combustion chamber of a downsized engine permits a better operation region that limits some typical engine losses such as pumping and friction losses, thus leading to higher thermal efficiency. This technique is normally implemented with high compression ratio and inlet gas charging in order to maintain power levels, which will lead to higher pressure and temperature at TDC (Top Dead Center), and thus resulting in great tendency of knock. Knock is unfavorable to engine thermal efficiency and, under severe conditions, causes erosion and damage of combustion chamber. It's commonly believed that knock is mainly caused by end gas auto-ignition, usually triggered by hot spots [2-4] which are formed through multiple ways, such as heat transfer from hot surface, convection with residual gas and temperature inhomogeneity due to turbulence, heat losses through walls and fuel injection. Besides, high peak pressure of pressure oscillation during super knock is considered by some researchers to be caused by the occurrence of detonation [5-8], no matter it is formed directly by hot spot or by acceleration of flame front.

Unlike the ignition triggered by spark, which usually develops into a subsonic deflagration, autoignition initialized from a hot spot with mild temperature gradient would results in much more intensive combustion mode, leading to pressure oscillation in combustion chamber. The different regimes of reaction wave initialized by hot spots with non-uniform initial conditions were first studied theoretically and numerically by Zeldovich [9] using a one-step chemical model. He figured out that spontaneous reaction wave can propagate through a reactive material along a spatial gradient of parameters such as temperature and species concentration that leads to different reactivities or ignition delay. Considering an area with temperature gradient along $\mathrm{x}$-direction, autoignition will first occur at the location with the temperature corresponding to the shortest ignition delay, and the spontaneous reaction wave then propagates along the gradient with the speed:

$u_{s p}=\left(\frac{\delta \tau_{i}}{\delta x}\right)^{-1}=\left(\frac{\delta \tau_{i}}{\delta T} \frac{\delta T}{\delta x}\right)^{-1}$

where $\tau_{i}$ is ignition delay as a function of initial temperature gained from experiment or chemical kinetics calculation. According to their relationships with Chapman-Jouguet speed and sound speed, different values of $u_{s p}$ indicate the initialization of different regimes of spontaneous reaction wave, such as detonation, deflagration and homogenous explosion. This gradient theory was afterwards studied by many researchers using detailed chemistry, who focused mainly on the initialization and propagation of reaction wave [10-13]. In multi-dimensional simulation, Robert et al. [14] studied knocking phenomenon in a downsized engine using LES and found that super-knock due to Deflagration to Detonation Transition (DDT) is triggered by coupling of auto-ignition front, while non-coupling of auto-ignition only causes conventional knock. DDT in hydrogen-air mixtures with concentration gradients numerically studied by Wang [15] shows that unburned hydrogen can be potentially re-ignited once more fresh air is available, resulting in subsequent explosions.

Based on these theory, initialization of flame front along hot spot with temperature gradient is closely linked to auto-ignition phenomenon. However, the effect of pressure wave on unburned mixture in closed space is rarely considered in simulation carried out before. No matter how autoignition behaves, pressure wave is always generated at the initial moment of combustion due to 
density discontinuity between burned and unburned area, and travels away from flame front at local sound speed if no detonation is formed [16]. Experiments also showed that pressure oscillation is caused by pressure wave [17], which compresses and disturbs unburned gas, creating turbulence and inhomogeneity, is closely linked to transition phenomenon of flame front. When in a closed chamber like engine cylinder, pressure wave could be reflected by cylinder walls and interacts with reaction front, thus making combustion process more complicated. Yu and Chen [18] simulated hydrogen/air mixture in a 1-D closed chamber, whose results showed that auto-ignition and detonation development can be induced by increasing the initial temperature, initial pressure, or chamber length. The similar effect of interaction between pressure wave and end gas is studied by Matsuura et al. [19], in which they found that a balance would be reached between flame speed and compression intensity to set back auto-ignition, which results in less chance for knock. Yamashita et al. [20] realized visualization observation of spontaneous ignition under controlled burst pressure in a plunger system, whose results confirmed that multiple auto-ignition points occurs near wall due to shock wave propagation in the tube. Oran and his co-workers [21] numerically studied shock-flame interactions in reactive mixture. They found that flame generates and enhances shock waves, which in turn creates turbulence in flames, thus promoting the formation of hot spots, causing autoignition and DDT. Similar phenomenon were also observed by Molkov et al. [22], but in a much larger scale. They modeled hydrogen-air deflagration in a long tunnel, where initial pressure wave develops into a shock due to reflection by obstacles.

From works mentioned above, auto-ignition under effect of pressure wave is supposed to be a possible cause of transition from one from to another, the investigation into which needs to be maintained. The aim of this work is to investigate formation of different modes of flame front triggered by auto-ignition of unburned mixture affected by propagation of pressure wave under ambient conditions near TDC. One dimensional simulation is carried out in an enclosed space with detailed chemistry. Different initial conditions are considered, within which flame front is initialized by hot spots. Several typical modes of flame development are analyzed and mass fraction of $\mathrm{OH}$ is employed to indicate the auto-ignition progress of unburned gas. One-dimensional simulation is conducted here because of its detailed description of pressure wave and flame structure and clarity of analysis. It has to be stated that this simulation rules out the effect of turbulence, boundary layer and heat transfer from walls, as well as inhomogeneity caused by intake and compression in practical engine. Besides, selection of initial temperature is slightly higher than in practical enginerelevant condition, in order to accelerate auto-ignition progress and reduce total calculation time to save computational resources.

\section{Specifications of model and numerical methods}

\subsection{Physical model}

As shown in Fig. 1, stoichiometric hydrogen/air mixture is filled in a one-dimensional domain with length $L=2 \mathrm{~cm}$, which is a good order of magnitude of the distance between the piston and the cylinder head a few crank angle degrees after Top Dead Center (TDC). Hydrogen, known as a renewable clean energy carrier, is utilized as fuel of SI engine in which abnormal combustion also occurs as in that fueled by gasoline [23-25]. Also, chemical mechanism of hydrogen is the most wellestablished and the concisest. Compared with simulation carried out with one-step model [12], 
detailed mechanism of hydrogen shows more accurate results in calculating low temperature reaction and spontaneous reaction wave, which is extremely crucial to this study. Therefore detailed hydrogen combustion mechanism of 9 species and 25 reactions, developed by Li et al. [26], is employed in this study, since it shows good performance in predicting ignition delay and flame speed under high pressure. A recent study on ability of various recent hydrogen combustion mechanisms under engine relevant conditions can also be found in Ref. [27]. As this study focuses on auto-ignition and transition behavior of reaction front in presence of pressure wave, initial temperature gradient of hot spot, which could lead to different regimes of flame, is not considered here. Therefore, the length of hot spot at left boundary is set as $L_{h}$, with uniform temperature $T_{h}$ and pressure $P_{0}$, while the temperature and pressure in unburned area are $T_{0}$ and $P_{0}$, respectively. Table 1 lists the initial conditions of the cases being calculated. It is obvious that different $P_{0}$ and $T_{0}$ mean different tendency of auto-ignition and different propagation speed of pressure wave and flame front. $P_{0}$ of $10 \mathrm{~atm}$ is low for unburned gas near TDC, but could exhibit more significant comparisons with cases of $40 \mathrm{~atm}$. As the role that pressure played in auto-ignition is minor compared with temperature, thus larger gap is needed to exhibit difference caused by pressure. Different $T_{h}$ indicates different ignition delay of hot spot, which results in different preparation time for end gas to auto-ignite prior arrival of flame front, however in this study only $1200 \mathrm{~K}$ is used since role of $T_{h}$ could be replaced by other parameters. Energy release within hot spot could be considered as instantaneous and the change of temperature distribution during ignition delay time could be neglected, thus all the mixture in hot spot is considered to auto-ignite simultaneously. Rapid heat release generates great pressure discontinuity between hot spot and unburned area, thus initializing a pressure wave propagating towards unburned mixture. Apparently, higher $L_{h}$ leads to stronger pressure wave, which diminishes slower than pressure wave initialized by that with lower $L_{h}$. The calculated cases exhibit quite different properties. Some typical cases are shown in details in the following sections. $T_{0}$ is set between $900 \mathrm{~K}$ and $1100 \mathrm{~K}$, which is slightly higher than unburned gas mixture in common SI (Spark Ignition) engine, to promote auto-ignition within relatively shorter period and smaller domain size. Actually, this temperature range can be easily achieved in a $\mathrm{HCCl}$ (Homogenous Charge Compression Ignition) engine [28] as well as in a $\mathrm{GCl}$ (Gasoline Compression Ignition) engine [29]. Moreover, inlet gas charging, fuel direct injection and temperature inhomogeneity due to above mentioned reasons may also result in local high temperature, therefore the selection of TO can be considered reasonable in this study.

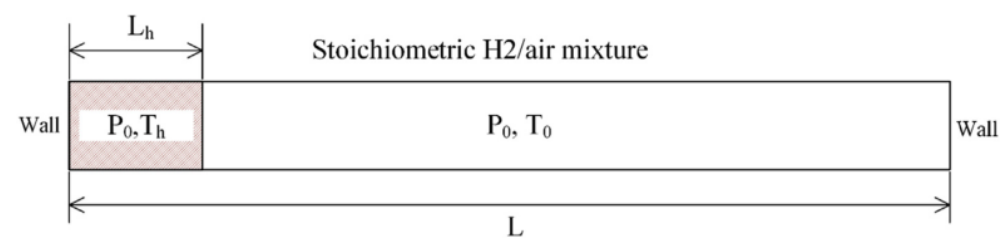

Figure 1 Schematic of boundary initial conditions in one-dimensional domain.

\begin{tabular}{lcccc}
\multicolumn{5}{l}{ Table 1 - Typical cases investigated in this study. } \\
\hline Case number & $\mathrm{L}_{h}$ & $\mathrm{~T}_{\mathrm{h}}$ & $\mathrm{P}_{0}$ & $\mathrm{~T}_{0}$ \\
\hline$(1)$ & $0.5 \mathrm{~mm}$ & $1200 \mathrm{~K}$ & $10 \mathrm{~atm}$ & $900 \mathrm{~K}$ \\
$(2)$ & $0.5 \mathrm{~mm}$ & $1200 \mathrm{~K}$ & $10 \mathrm{~atm}$ & $1100 \mathrm{~K}$ \\
$(3)$ & $5 \mathrm{~mm}$ & $1200 \mathrm{~K}$ & $10 \mathrm{~atm}$ & $1100 \mathrm{~K}$ \\
$(4)$ & $0.5 \mathrm{~mm}$ & $1200 \mathrm{~K}$ & $40 \mathrm{~atm}$ & $1100 \mathrm{~K}$ \\
\hline
\end{tabular}




\section{$2.2 \quad$ Numerical methods}

Simulation is carried out using in-house code Adaptive Simulation of Unsteady Reactive Flow (ASURF), which showed good performance in recent works [30-32] for predicting auto-ignition and propagation of flame front and pressure wave. The code solves the conservation equations of one dimensional, compressible, multi-component, reactive flow using finite volume method:

$\frac{\delta U}{\delta t}+\frac{\delta F(U)}{\delta x}=F_{v}(U)+S_{R}$

where the vectors $U, F(U), F_{v}(U)$, and $S_{R}$ are defined as:

$U=\left(\begin{array}{c}\rho Y_{1} \\ \rho Y_{2} \\ \vdots \\ \rho Y_{N S} \\ \rho u \\ E\end{array}\right), F(U)=\left(\begin{array}{c}\rho u Y_{1} \\ \rho u Y_{2} \\ \vdots \\ \rho u Y_{N S} \\ \rho u^{2}+P \\ (E+P) u\end{array}\right), F_{v}(U)=\left(\begin{array}{c}-\rho Y_{1} V_{1}^{\prime} \\ -\rho Y_{2} V_{2}^{\prime} \\ \vdots \\ -\rho Y_{N S} V_{n}^{\prime} \\ \tau \\ q\end{array}\right), S=\left(\begin{array}{c}\omega_{1} \\ \omega_{2} \\ \vdots \\ \omega_{n} \\ 0 \\ 0\end{array}\right)$

where $\rho$ denotes the density, $u$ the velocity, $E=-P+\frac{\rho u^{2}}{2}+\rho h$ the total energy per unit volume, $h$ the total enthalpy per unit volume, $P$ the hydrostatic pressure, $\tau$ the viscous stress tensor, $q$ the heat flux, $Y_{k}, V_{k}^{\prime}$ and $\omega_{k}$ the mass fraction, diffusion velocity vector and production rate of the $k$ th species, respectively.

Strang splitting fractional-step procedure of second-order accuracy is utilized to separate time evolution of the stiff reaction term from that of the convection and diffusion terms. In the first fractional step, the non-reactive flow is solved. The Runge-Kutta, central difference, and MUSCLHancock schemes, all of second-order accuracy, are employed for calculating temporal integration, diffusive flux and convective flux, respectively. In the second fractional step, detailed chemistry is solved using VODE solver, while reaction rates, thermodynamic and transport properties are evaluated using CHEMKIN and TRANSPORT packages [33,34]. A multi-level, dynamically adaptive mesh refinement algorithm is used to maintain adequate numerical resolution of moving reaction and pressure front. When solving problems where shock wave occurs, proper mesh resolution is quite crucial to obtain a result within minor error while cost acceptable computing resources. In considering both accuracy and computational time, the flame front and pressure wave are eventually covered by the finest mesh of $0.4 \mu \mathrm{m}$ by adjusting CFL number, with corresponding time step of 5 e-11 s. Previous works $[13,18]$ also showed that A-SURF can accurately capture shock wave propagation and show good prediction of detonation speed close to that of Chapman-Jouguet value. More details of numerical schemes and code validations of A-SURF can be found in Refs. $[35,36]$ and Supplementary Materials.

\section{Results and discussion}

\subsection{Indicator of auto-ignition progress}

Several parameters were used to indicate reaction progress of unburned mixture, for instance, temperature of end gas [37] and value of Livengood-Wu Integral [38,39]. However, during simulation 
of this work, temperature of unburned mixture is affected not only by heat release of reaction, but also by compression and expansion effect of pressure wave, which makes temperature impractical to be an accurate indicator. Livengood-Wu Integral enables prediction of ignition delays under variable conditions, but is only supposed to be used without detailed chemistry where low temperature reaction is ignored. In that case, the mass fractions of specific intermediate species in pre-mixed unburned gas which are only affected by chemical reactions and species transport tend to be a proper parameter to represent auto-ignition progress. A chemical kinetic study is carried out using CHEMKIN-PRO to investigate the capability of species mass fraction of indicating auto-ignition progress, where ignition moment is defined as the moment with maximum temperature rise rate. Fig. 2 shows the evolutions of mass fractions of intermediate species during zero-dimensional homogenous auto-ignition of hydrogen/air mixture. It is seen that mass fractions of $\mathrm{H}, \mathrm{O}$ and $\mathrm{OH}$ increase rapidly near auto-ignition moment, while mass fractions of $\mathrm{HO}_{2}$ and $\mathrm{H}_{2} \mathrm{O}_{2}$ increase to a considerable amount at early stage but change slowly near auto-ignition moment compared with that of $\mathrm{H}, \mathrm{O}$ and $\mathrm{OH}$. Thus, $\mathrm{OH}$ is selected as the indication species for its considerable growth rate and amount during ignition delay time. The evolution of mass fraction of $\mathrm{OH}, Y_{\mathrm{OH}}$, under different initial conditions are shown in Fig. 3. It can be observed that $\mathrm{YOH}$ reaches peak value near ignition moment and stays at a lower value after chemical equilibrium is reached. Since $Y_{\mathrm{OH}}$ at ignition moment differs from each other for different initial conditions, a threshold value exceeding which the ignition is excited, is to be decided for indication of auto-ignition progress. $Y_{\mathrm{OH}}=1 e-4$ seems to be an ideal selection as unburned mixture at different cases all come to ignition within $1.5 \mu$ s after threshold value is reached and maximum difference between each case is less than $1 \mu$ s Autoignition progress, $I$, is therefore defined as normalized mass fraction of $\mathrm{OH}: I=Y_{\mathrm{OH}} / 1 e-4$. When $I$ reaches unity, auto-ignition is excited and would occur within several microseconds. If mixture at one location is burned due to arrival of flame front, $I$ would suffer sharp increment and reaches a value much higher than unity within very short time interval since intermediate species have very few time to accumulate. Therefore, with indication of I, whether combustion is due to flame propagation or auto-ignition can be inferred, and the extent how unburned mixture is close to autoignition can be qualitatively perceived.

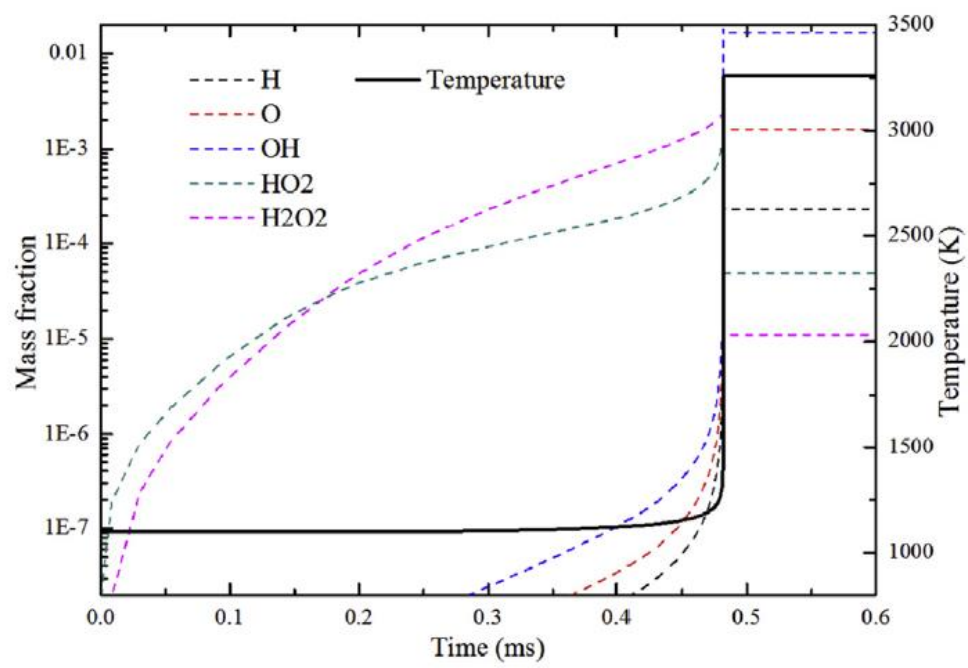

Figure 2 Evolutions of mass fractions of intermediate species during homogenous auto-ignition of stoichiometric hydrogen/air mixture with $P_{0}=40 \mathrm{~atm}, T_{0}=1100 \mathrm{~K}$. 


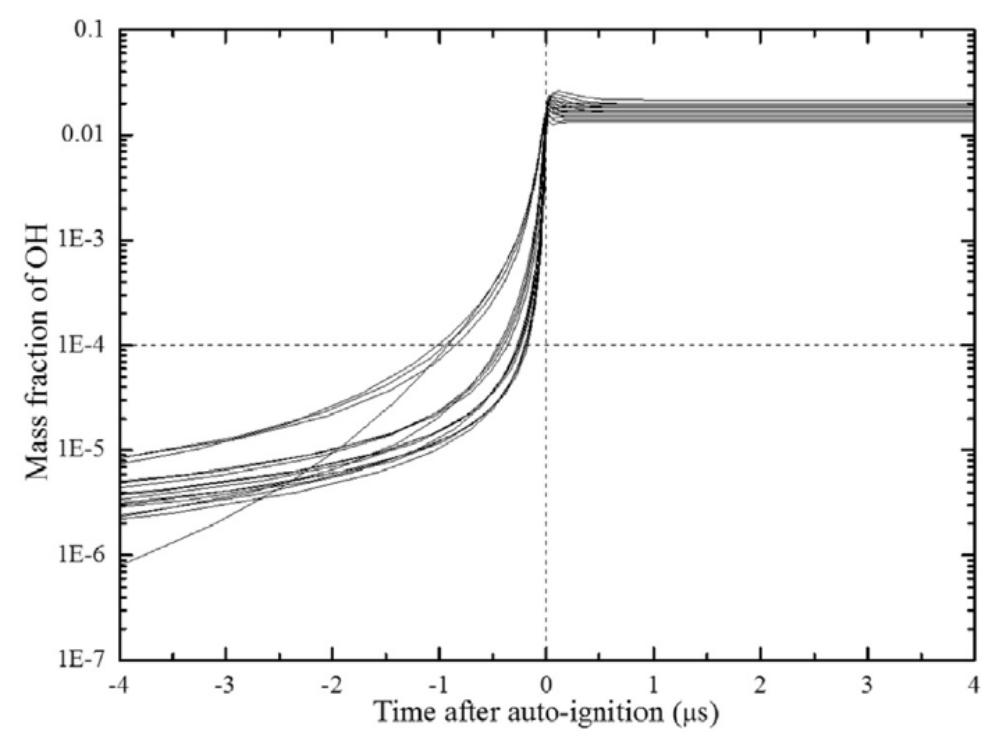

Figure 3 Evolutions of YOH under different initial conditions with: $P_{0}=10,20,30,40$ atm and $T_{0}=900,1000,1100$, $1200 \mathrm{~K}$.

\subsection{Developing detonation}

Results obtained from Case (1) is observed with absence of auto-ignition, the reason of which is expected to be insufficient temperature of unburned mixture to reach ignition delay before arrival of flame front. Therefore the results of Case (1) are not shown here. In order to achieve end gas autoignition before main flame consumes all mixture, $T_{0}$ has to be increased so that ignition delay of end gas decreases to a level shorter than propagation time of flame front. Fig. $\mathbf{4}$ shows evolutions of temperature and pressure profiles in Case (2). Compared with Case (1), unburned mixture attains sufficiently high reactivity to reach auto-ignition before initial flame front sweeps through entire domain. It can be observed that auto-ignition occurs at both reaction front and right boundary. At first, initial flame front propagates at the speed of $70 \mathrm{~m} / \mathrm{s}$ with pressure wave propagating at 900 $\mathrm{m} / \mathrm{s}$, which approximately equals to sound speed. The transition of reaction front occurs at 118.35 $\mu \mathrm{s}$, at which moment the pressure profile comes up with a sudden rise. This explosion-like phenomenon generates two pressure waves, one propagating towards the same direction as the reaction front with very high peak value and sharp discontinuity, while the other propagating towards the opposite direction but with a much lower peak value, which is expected to be caused by partially transmissive effect of reaction front, just as mentioned in Case (1). The right-propagating pressure wave couples with flame front and propagates together at the speed of approximately $1957 \mathrm{~m} / \mathrm{s}$, which is very close to the Chapmane-Jouguet (C-J) detonation speed of $1958 \mathrm{~m} / \mathrm{s}$. It has to be noted that C-J speed here is calculated based on $T_{0}$ and $P_{0}$, which would be slightly different from that based on conditions of unburned gas affected by pressure wave and slow reaction. The mixture at right boundary auto-ignites a few microseconds earlier than the occurrence of transition and forms a detonation at the speed around $2047 \mathrm{~m} / \mathrm{s}$, which decelerates to approximately $1930 \mathrm{~m} / \mathrm{s}$, nearly the same as that formed by flame front transition. Same transition process is also observed in the works of $\mathrm{Yu}$ and Chen [18], in which the author suggested that the transition is mainly due to local auto-ignition in front of flame front. In order to figure out if the auto-ignition occurs, $I$ at transition location $x=0.72 \mathrm{~cm}$, as well as that at $x=1.20 \mathrm{~cm}$ and $x=1.99 \mathrm{~cm}$, are investigated. 


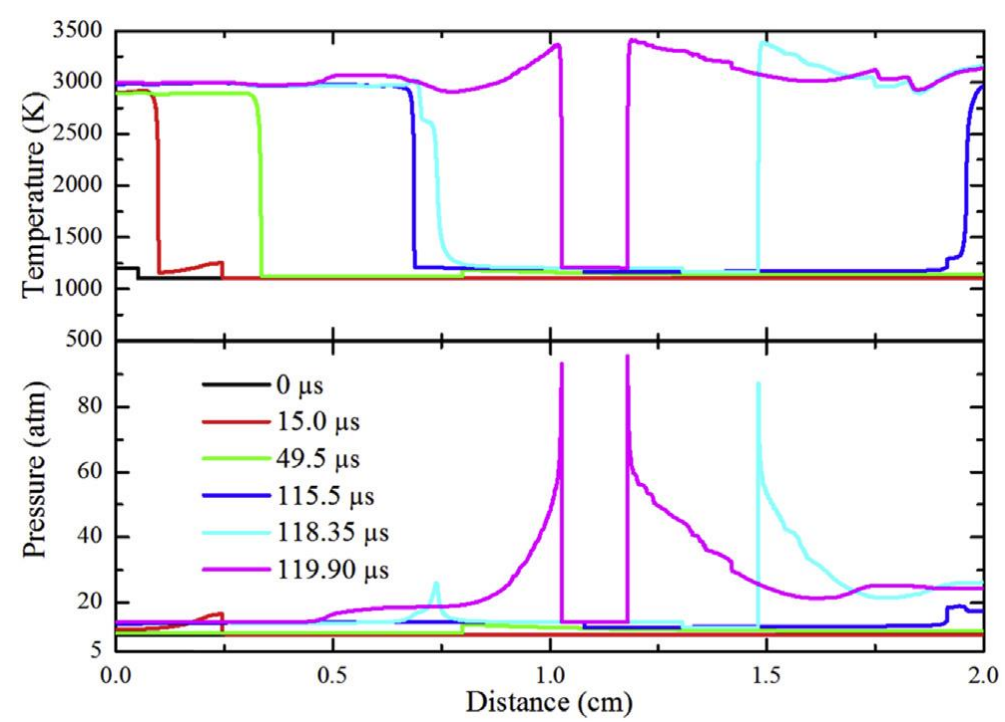

Figure 4 Evolutions of temperature and pressure profiles in Case (2).

As unburned gas mixture is affected by propagating pressure wave and would not stay at one location, particles tracking method is implemented to acquire parameters that varies as location of particles are influenced by pressure wave. In simulation, the position of a tracking particle is first updated according to its flow velocity and time step size, then its thermodynamic conditions, flow velocity and species fraction are obtained implementing linear extrapolation of corresponding values at its two neighboring grids. Evolutions of temperature and I at different locations are plotted in Fig. 5. It can be observed that temperature at different locations oscillates as pressure wave passes by, while $x=1.99 \mathrm{~cm}$ suffers the highest temperature increase due to reflection of pressure wave. The three locations are affected by pressure wave several times, each of which increases the value of $I$ due to chemical equilibrium at different temperature, making it faster for this location to reach autoignition, although value of $I$ decreases a little as temperature decreases after passage of pressure wave. Comparing $I$ at $x=0.72 \mathrm{~cm}$ with $x=1.99 \mathrm{~cm}$, it can be seen that pressure wave causes 3 times more increases of $I$ as it passes through $x=0.72 \mathrm{~cm} 5$ times regardless of propagation direction, while pressure wave can only be reflected at $x=1.99 \mathrm{~cm}$. However, temperature rise caused by pressure wave reflection is higher, therefore the auto-ignition progress indicated by $I$ at $x=1.99 \mathrm{~cm}$ exceeds that at $x=0.72 \mathrm{~cm}$ at approximately $35 \mu$ After several interactions with pressure wave, unburned gas in front of flame front and at right boundary finally reaches critical condition of auto-ignition, with temperature rising sharply above $2000 \mathrm{~K}$ and $I$ reaching 1.0 . In comparison, $I$ at $x=1.20 \mathrm{~cm}$ reaches only 0.017 before this location is swept by flame, and rapidly increases to more than 100 due to combustion triggered by detonation front, which indicates the absence of auto-ignition. 


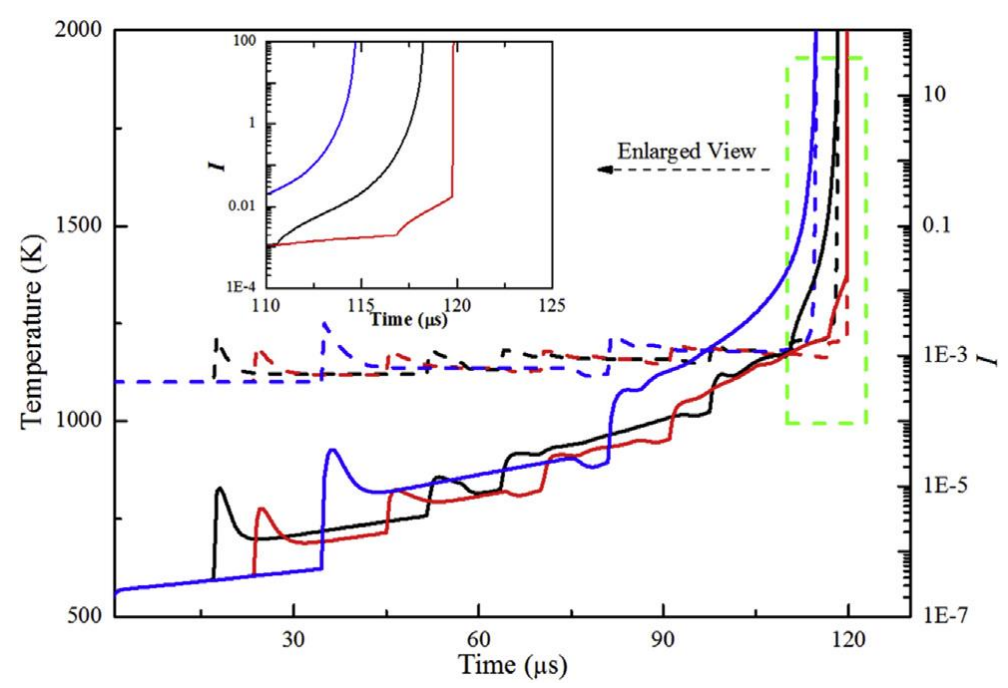

Figure 5 Evolutions of temperature and $I$ at different locations. Dotted lines represent temperature profiles and solid lines represent I profiles. Location sequences are: black $-x=0.72 \mathrm{~cm}$, red $-x=1.20 \mathrm{~cm}$, blue $-x=1.99 \mathrm{~cm}$. (For interpretation of the references to colour in this figure legend, the reader is referred to the web version of this article.)

To further verify that transition is triggered by local high reactivity, auto-ignition progress near transition location is investigated. Fig. 6 shows evolutions of temperature and $I$ near $x=0.72 \mathrm{~cm}$. It can be seen that transition process starts near $118.00 \mu$ s and a stable detonation wave with strong discontinuity is formed at about $119.00 \mu \mathrm{s}$. The profile of $I$ at both $118.00 \mu$ s and $118.50 \mu$ s remain smooth and continuous ahead of flame front, which ranges from 0.1 to 10 , indicating that the unburned mixture along a section of distance with temperature gradient near flame front is very close to auto-ignition. In comparison, profile of $I$ at $119.00 \mu$ s has obvious discontinuity at $x=0.85$ $\mathrm{cm}$ through which $I$ jumps from 0.6 to 300 that evades the range close to auto-ignition, which is due to discontinuity of detonation wave. Thus unburned mixture is ignited by intense precursor shock of detonation, rather than auto-ignition ahead of flame front due to self-reinforced chemical reaction at $118.00 \mu \mathrm{s}$ and $118.50 \mu \mathrm{s}$.

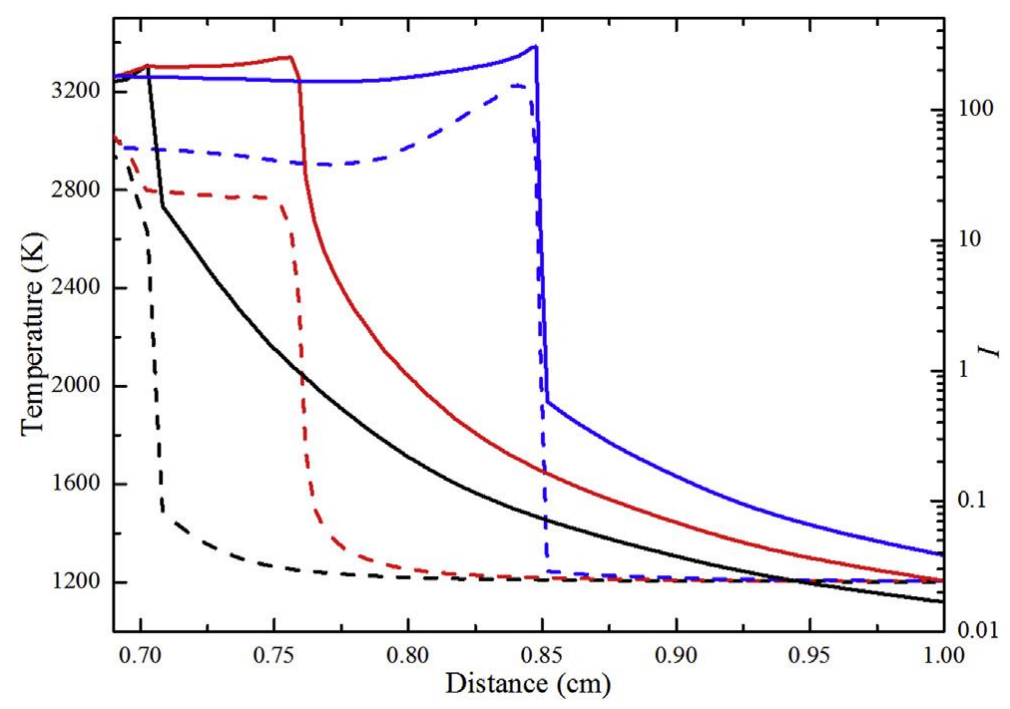

Figure 6 Evolutions of temperature and $I$ near $x=0.72 \mathrm{~cm}$ in Case (2). Dotted lines represent temperature profiles and solid lines represent $I$ profiles. Time sequences are: black $-\mathbf{1 1 8 . 0 0} \mu \mathrm{s}$, red $-\mathbf{1 1 8 . 5 0} \mu \mathrm{s}$, blue $-\mathbf{1 1 9 . 0 0} \mu \mathrm{s}$. (For interpretation of the references to colour in this figure legend, the reader is referred to the web version of this article.) 


\subsection{Effect of pressure wave on DDT}

If pressure wave is strong enough, unburned mixture would be heated up to auto-ignition very fast, which is similar to direct detonation from a hot spot, and end gas auto-ignition has no sufficient time to occur before flame front arrives as detonation is formed at very early stage. Fig. 7 shows the evolutions of temperature and pressure profiles in Case (3). As $L_{h}$ increases compared with Case (2), more energy is released when hot spot ignites and therefore a stronger pressure wave is generated, with overpressure of about 7.5 bar, which is higher than that in Case (2). The pressure rise caused by this stronger pressure wave also lasts longer, which maintains high temperature for longer period. The more effective impact of pressure wave accelerates auto-ignition process ahead of flame front, which finally forms a detonation at early stage. The detonation propagates near C-J detonation speed, quickly catches up with initially formed pressure wave and arrives right boundary without occurrence of end gas auto-ignition.

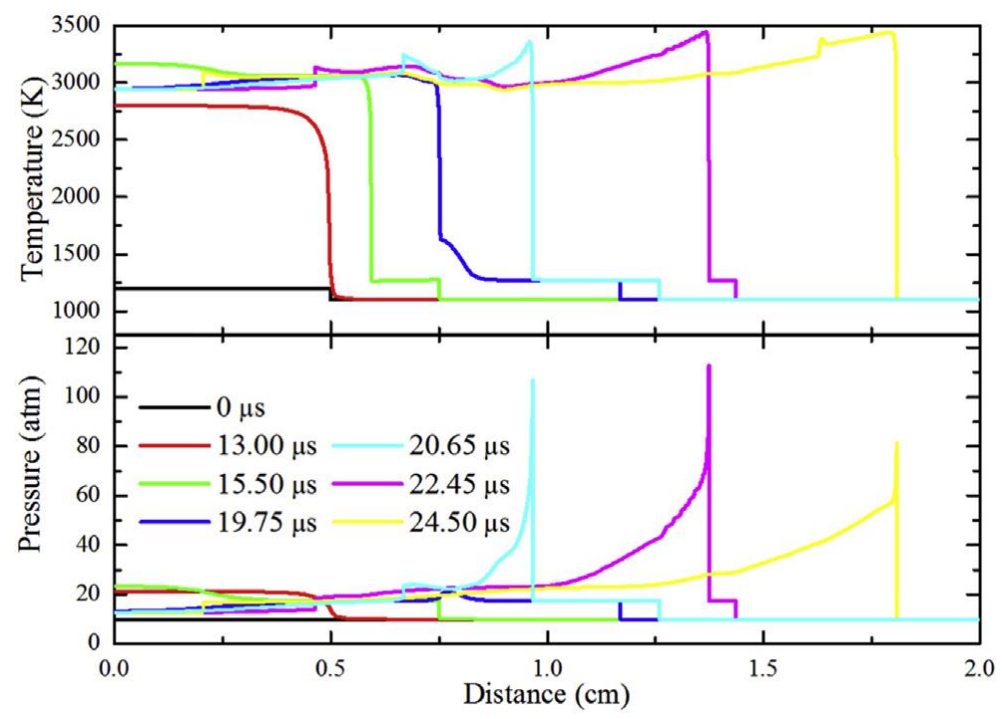

Figure 7 Evolutions of temperature and pressure profiles in Case (3).

Evolutions of temperature and I near transition location $x=0.8 \mathrm{~cm}$ are plotted in Fig. 8 . It can be observed that leading pressure wave compresses unburned mixture near $x=1.20 \mathrm{~cm}$ and causes temperature rise, which significantly leads I to a considerable value that continues to increase before flame front arrives. The initial deflagration propagates into highly-reactive unburned mixture and encounters similar transition process with that in Case (2), however the high reactivity of unburned gas ahead of flame front is mainly induced by stronger pressure wave rather than both weaker pressure wave and accumulation of intermediate species. Since propagation speed of detonation is quite high, the remaining unburned gas has no sufficient time to come to auto-ignition before detonation arrives, as the discontinuity which can be observed in profile of $20.50 \mu$ s. 


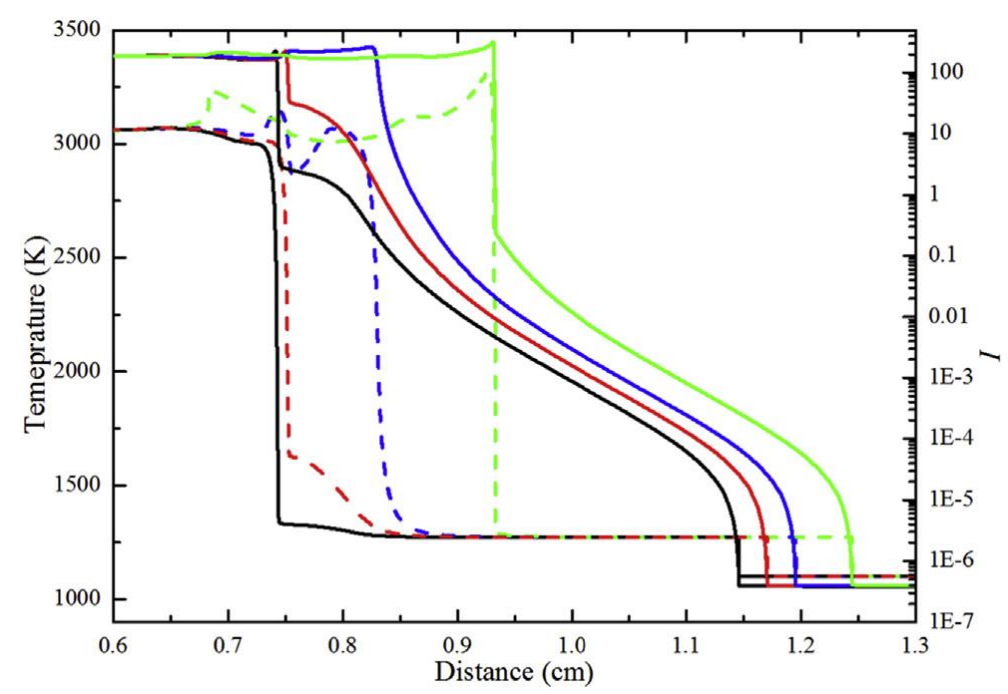

Figure 8 Evolutions of temperature and I near $x=0.8 \mathrm{~cm}$ in Case (3). Dotted lines represent temperature profiles and solid lines represent $I$ profiles. Time sequences are: black $-19.50 \mu \mathrm{s}$, red $-19.75 \mu \mathrm{s}$, blue $-20.00 \mu \mathrm{s}$, green -20.50 $\mu \mathrm{s}$. (For interpretation of the references to colour in this figure legend, the reader is referred to the web version of this article.)

\subsection{Supersonic deflagration}

In above sections, transition from deflagration to detonation is observed and can be attributed to high reactivity of unburned gas ahead of flame front. Once detonation is formed, unburned mixture is rapidly swept by it because detonation leaves no sufficient time. However, there still exists possibility that $I$ ahead of detonation front reaches a sufficient high level that unburned mixture comes to auto-ignition before caught up by detonation. In that consideration, another transition mode from detonation to supersonic auto-ignitive deflagration is simulated through Case (4). With higher $P_{0}$, the ignition delay of hot spot is longer and the propagation speed of flame front is slower, which leaves more preparation time for auto-ignition and transition compared with Case (2). Fig. 9 shows evolutions of temperature and pressure profiles in Case (4). After a period of time during which the flame front and pressure wave propagates, end gas auto-ignition occurs at right boundary and develops into a detonation propagating near C-J detonation speed at $221.50 \mu \mathrm{s}$, until $223.35 \mu \mathrm{s}$, the unburned mixture in front of left-propagating detonation begins to auto-ignite. The auto-ignition forms a new reaction front propagating at a speed of above $5000 \mathrm{~m} / \mathrm{s}$, which is much higher than C-J detonation speed. As new flame front decouples from initial pressure wave, the pressure peak of leading pressure wave decreases from about 374 atm to less than 244 atm, but still higher than that of $104 \mathrm{~atm}$ behind detonation wave. The new flame front after transition of initial detonation wave could be considered as a supersonic deflagration. Obviously, the auto-ignition progress of the location in front of detonation wave reaches a critical value, which leaves no sufficient time for the arrival of detonation. As auto-ignition progress of location closer to initial reaction front is faster, it auto-ignites earlier than the location, which is further. In this sequential auto-igniting manner, a deflagration is formed but propagates at a much faster speed than detonation does. 


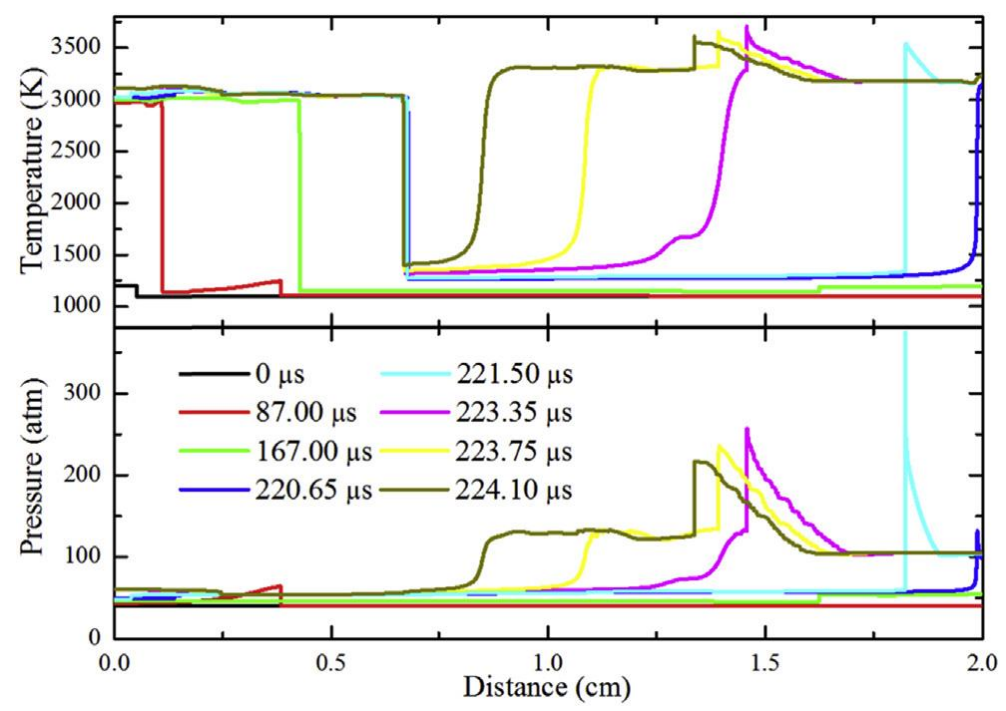

Figure 9 Evolutions of temperature and pressure profiles in Case (4).

The temperature and $I$ profiles of the three representative locations in Case (4) are plotted in Fig. 10. Three tracked locations are $x=1.39 \mathrm{~cm}, x=1.70 \mathrm{~cm}$ and $x=1.99 \mathrm{~cm}$, representing locations that are swept by supersonic deflagration, detonation and auto-ignition, respectively. It can be seen from temperature profiles that pressure wave generated by main flame front propagates through three locations at different times and causes pressure oscillations leading to different temperature rise. The same as shown in Fig. 4, the right boundary $x=1.99 \mathrm{~cm}$ suffers the greatest temperature rise due to reflection of pressure wave, which greatly speeds up its auto-ignition progress. The $I$ at $x=1.99 \mathrm{~cm}$ is the first to reach unity, indicating the first occurrence of auto-ignition at $x=1.99 \mathrm{~cm}$. Then I at $x=1.70 \mathrm{~cm}$ jumps from 0.1 to 100 at about $221.75 \mu$ s due to arrival of detonation wave from right boundary. Even though $x=1.39 \mathrm{~cm}$ is swept by deflagration front, I profile of it smoothly increases from 0.1 to 10 without sharp discontinuity, indicating that auto-ignition appears at this location. Evolutions of temperature and $I$ near $x=1.39 \mathrm{~cm}$ are plotted in Fig. 11. When detonation is formed and propagating towards left at $221.50 \mu \mathrm{s}, I$ ahead of detonation front approaches 0.1 , which is quite close to auto-ignition. Auto-ignition progress keeps increasing as shown by profile of $223.00 \mu \mathrm{s}$. It can be observed that transition starts around $223.30 \mu$ s while $I$ ahead of detonation front exceeds 1. Unlike in Figs. $\mathbf{6}$ and 8, I profile in Fig. $\mathbf{1 1}$ not only has higher overall value, but also has milder gradient, which means the unburned mixture would auto-ignite earlier and the ignition moments of different locations are much more close. 


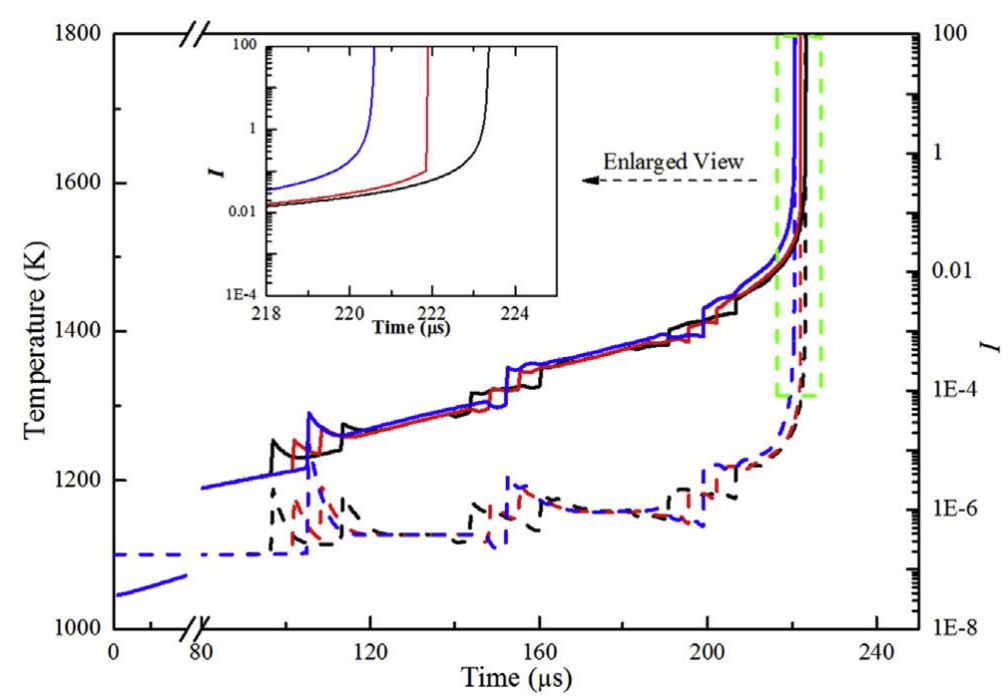

Figure 10 Evolutions of temperature and $I$ at different locations in Case (4). Dotted lines represent temperature and solid lines represent $I$. Location sequences are: black $-x=1.39 \mathrm{~cm}$, red $-x=1.70 \mathrm{~cm}$, blue $-x=1.99 \mathrm{~cm}$. (For interpretation of the references to colour in this figure legend, the reader is referred to the web version of this article.)

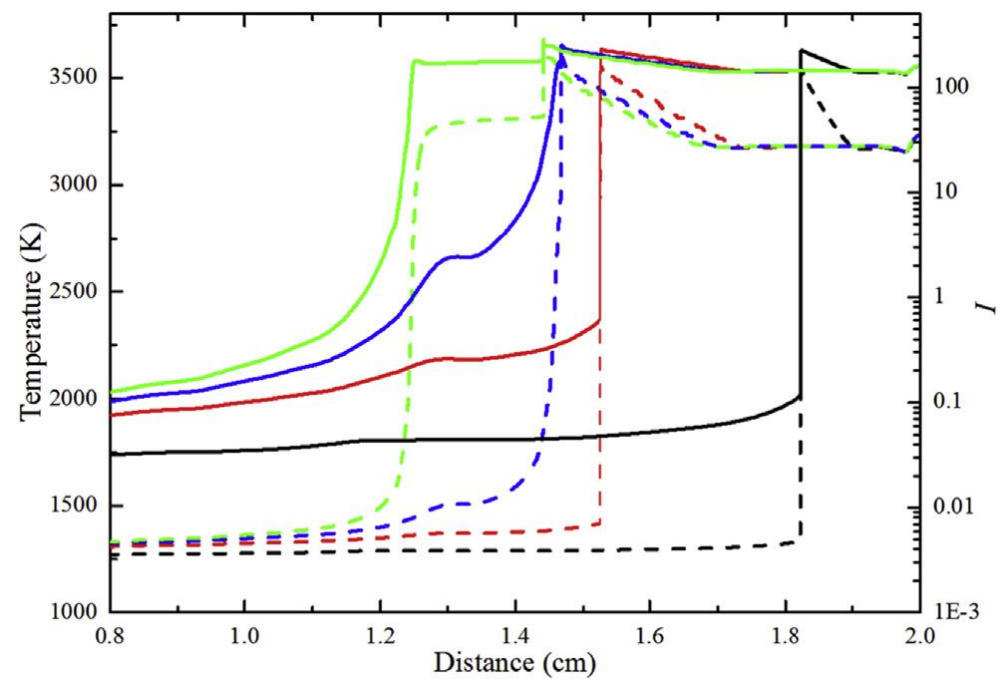

Figure 11 Evolutions of temperature and $I$ near $x=1.39 \mathrm{~cm}$ in Case (4). Dotted lines represent temperature profiles and solid lines represent $I$ profiles. Time sequences are: black $-221.50 \mu \mathrm{s}$, red $-223.00 \mu \mathrm{s}$, blue $-223.30 \mu \mathrm{s}$, green

$-223.45 \mu \mathrm{s}$. (For interpretation of the references to colour in this figure legend, the reader is referred to the web version of this article.)

Since the condition of unburned gas is changing due to propagating pressure wave, it is difficult to give an exact prediction of time interval from one auto-ignition progress to another. However, the transition mechanism between detonation and supersonic deflagration, as well as the role played by gradient, can be further demonstrated through one simple simulation similar to the work of Kapila et al. [40]. As shown in Fig. 12a, a two-stage linear temperature gradient is introduced in calculation domain. Temperature first decreases from $1800 \mathrm{~K}$ at left boundary to $1240 \mathrm{~K}$ at $2.25 \mathrm{~cm}$, and then to $1200 \mathrm{~K}$ at right boundary, while $P_{0}=10 \mathrm{~atm}$ and total length of $4 \mathrm{~cm}$. It can be observed that a supersonic deflagration is first formed near left boundary and then transits into detonation near $t=4.50 \mu \mathrm{s}$. The reason is expected to be coupling between shock and reaction zone caused by decrease of flame front. The detonation propagates for a short distance until a new reaction front, which is actually a supersonic deflagration front, is formed in front of detonation front near $t=8.00$ $\mu s$. Finally, before flame reaches right boundary, supersonic deflagration again transits into 
detonation, which is indicated by the second pressure peak at $t=12.20 \mu \mathrm{s}$. Evolutions of flame speed, $u_{s p}$ calculated by Eq. (1) and $u_{1 D}$ simulated by ASURF simulation, are plotted in Fig. 12b to clarify this complex transition process. It is obvious that $u_{1 D}$ matches well with $u_{s p}$ at the first stage and maintains near C-J speed $u_{C J}$ after $u_{s p}$ drops below $u_{1 D}$. However, $u_{1 D}$ does not exceed $u_{C J}$ near $L=2.34 \mathrm{~cm}$ as usp behaves, but is delayed until detonation transits back into supersonic deflagration near $L=2.74 \mathrm{~cm}$, which can be explained as that the time unburned gas goes through before reaction wave arrives is shorter than its $\tau_{i}$, which holds back auto-ignition ahead of reaction front. $\tau_{i}$ at $L=2.34 \mathrm{~cm}$ and $L=2.74 \mathrm{~cm}$ are $6.76 \mu \mathrm{s}$ and $7.84 \mu \mathrm{s}$, respectively. Finally, $u_{s p}$ again drops below $S_{C J}$, and supersonic deflagration transits into detonation again.

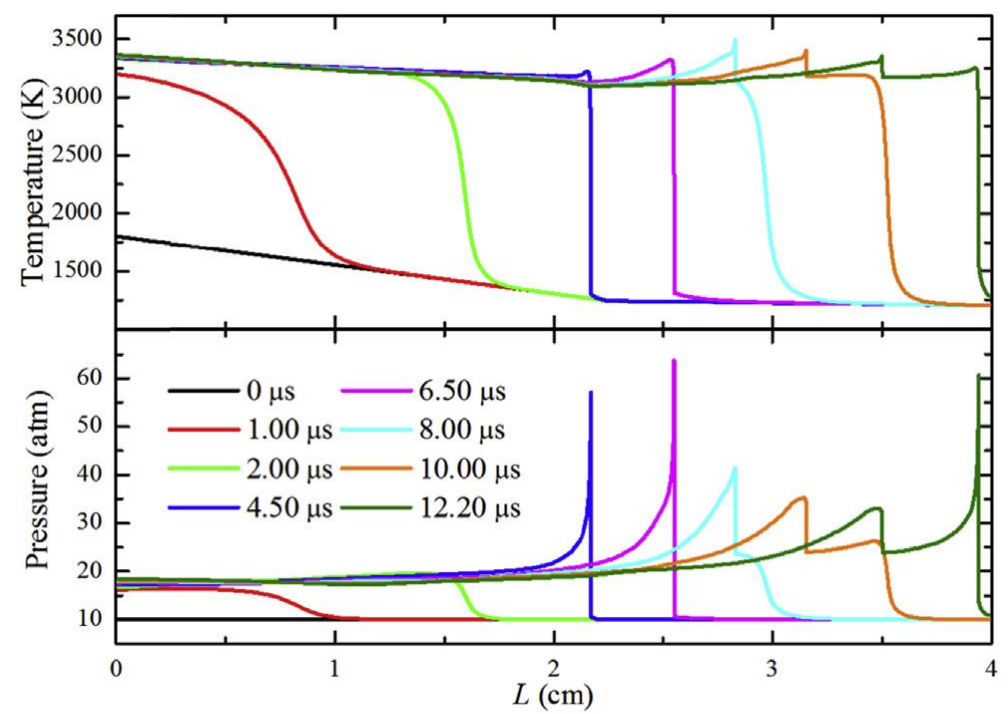

(a)

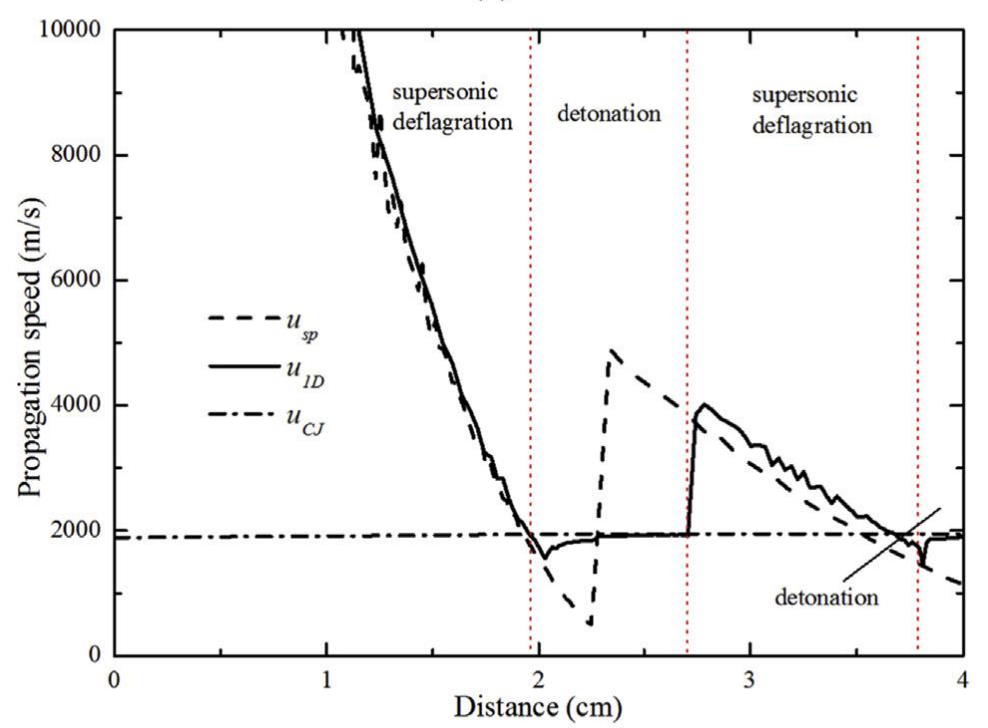

(b)

Figure 12 Transition between detonation and supersonic deflagration along a two-stage temperature gradient. (a) Evolutions of temperature and pressure profiles. (b) Propagation speed calculated by Eq. (1) and ASURF code. 


\section{Conclusions}

In this study, end gas auto-ignition and flame front transition of stoichiometric hydrogen/air mixture under effect of pressure wave is simulated in one-dimensional domain with detailed chemistry. Cases under different initial conditions are considered and different combustion modes due to pressure wave and auto-ignition are observed. The main findings can be summarized as follows:

During chemical kinetic study of homogenous auto-ignition of stoichiometric hydrogen/air mixture, mass fraction of $\mathrm{OH}$ is employed as indicator of auto-ignition progress and a threshold value of $1 e-4$ is found to be a proper criterion to identify auto-ignition moment with minor error less than $1.5 \mu$ s. When $I$ reaches unity at one location in ASURF simulation, auto-ignition is considered to occur.

Flame front is observed to exhibit different forms during propagation. As initial temperature increases, auto-ignition occurs at right boundary due to more intense higher reactivity of unburned mixture, while longer length of hot spot generates pressure wave with higher amplitude that triggers DDT at early stage of simulation. Supersonic deflagration is also observed in case with higher initial pressure, since longer ignition delay as well as lower propagation speed of flame front under higher pressure leaves unburned mixture more time to auto-ignite. With indication of $I$, transition of propagating flame front from deflagration to detonation is found to be triggered by local autoignition ahead of flame front.

Study on distribution of $I$ indicates that sequential auto-ignition is a major factor of transition of flame. Inhomogeneity is caused by inducement of pressure wave and lead to gradient distribution of $I$ along unburned mixture. When $I$ ahead of subsonic deflagration flame front reaches sufficiently high level with sharp gradient, transition from deflagration to detonation is supposed to happen. The detonation from right boundary is also transited from subsonic deflagration generated by end gas auto-ignition, but appears faster as end gas has higher $I$ due to reflection of pressure wave. If $I$ profile ahead of detonation has sufficiently high level with mild gradient, detonation starts to transit to supersonic deflagration. If end gas is not affected by pressure wave before flame arrives, no gradient of $I$ is formed and thermal explosion is about to happen after ignition delay time like a homogenous auto-ignition.

In general, this work discusses the method of utilizing $\mathrm{OH}$ species to indicate the auto-ignition progress of unburned gas and testifies the possible reason that leads to flame transition. It is similar to the works based on Zeldovich gradient theory but includes the influence of pressure wave on unburned gas conditions and more focuses on transition processes. The research shows some new aspects of interaction between flame propagation and auto-ignition, and might bring some new ideas on study of mechanism of knock and super-knock.

There are some limitations in this work that can be improved in future works. In order to limit the issues to a relatively simple view, one-dimensional simulation carried out here ignores the influence of turbulence, boundary layer, obstacles, heat transfer with walls, triple-point of shockwave and so on [41-44], which are believed to have non-ignorable effect on temperature distribution and occurrence of auto-ignition. 


\section{Acknowledgements}

The work is supported by National Natural Science Foundation of China (Grant Nos. 91641203, 51476114, 51606133).

\section{Appendix A. Supplementary data}

Supplementary data related to this article can be found at http://dx.doi.org/10.1016/i.ijhydene.2017.05.085

\section{References}

[1] Tim L, John S, Richard M, Richard O, Andreas S. Turbocharging concepts for downsized di gasoline engines. SAE technical paper, 2004-01-0036. 2004.

[2] Heywood JB. Internal combustion engine fundamentals. New York: McGraw-Hill College; 1988.

[3] Bradley D, Kalghatgi GT, Golombok M, Yeo J. Heat release rates due to autoignition, and their relationship to knock intensity in spark ignition engines. Symp (Int) Combust 1996;26:2653-60.

[4] Pan J, Shu G, Wei H. Interaction of flame propagation and pressure waves during knocking combustion in spark ignition engines. Combust Sci Technol 2014;186:192-209.

[5] Curry S. A three-dimensional study of flame propagation in a spark ignition engine. SAE technical paper, 1963-04-87. 1963.

[6] Qi Y, Wang Z, Wang J, He X. Effects of thermodynamic conditions on the end gas combustion mode associated with engine knock. Combust Flame 2015;162:4119-28.

[7] Wang Z, Qi Y, He X, Wang J, Shuai S, Law CK. Analysis of pre-ignition to super-knock: hotspotinduced deflagration to detonation. Fuel 2015;144:222-7.

[8] Wei H, Shang Y, Chen C, Gao D, Feng D. One-dimensional numerical study on pressure waveflame interaction and flame acceleration under engine-relevant conditions. Int J Hydrogen Energy 2015;40:4874-83.

[9] Zeldovich YB. Regime classification of an exothermic reaction with non-uniform initial conditions. Combust Flame 1980;39:211-4.

[10] Bradley D, Morley C, Gu XJ, Emerson DR. Amplified pressure waves during auto-ignition: relevance to CAl engines. SAE technical paper, 2002-01-2868. 2002.

[11] Gu XJ, Emerson DR, Bradley D. Modes of reaction front propagation from hot spots. Combust Flame 2003;133:63-74.

[12] Liberman MA, Kiverin AD, Ivanov MF. Regimes of chemical reaction waves initiated by nonuniform initial conditions for detailed chemical reaction models. Phys Rev E 2012;85:056312. 
[13] Peng D, Zheng C, Shiyi C, Yiguang J. Numerical experiments on reaction front propagation in $n$ heptane/air mixture with temperature gradient. Proc Combust Inst 2014;35:3045-52.

[14] Robert A, Richard S, Colin O, Poinsot T. LES study of deflagration to detonation mechanisms in a downsized spark ignition engine. Combust Flame 2015;162:2788-807.

[15] Wang CJ, Wen JX. Numerical simulation of flame acceleration and deflagration-to-detonation transition in hydrogen-air mixtures with concentration gradients. Int J Hydrogen Energy 2017;42:7657-63.

[16] Pan J, Sheppard C. A theoretical and experimental study of the modes of end gas auto-ignition leading to knock in S. I. engines. SAE technical paper, 942060. 1994.

[17] Hettinger A, Kulzer A. A new method to detect knocking zones. SAE Int J Engines 2009;2:645-65.

[18] $\mathrm{Yu} \mathrm{H}$, Chen Z. End-gas autoignition and detonation development in a closed chamber. Combust Flame 2015;162:4102-11.

[19] Matsuura K, Nakano K, Shimizu K, lida N, Sato Y. Effect of heat release pattern of flame during propagation on auto-ignition process of end gas. SAE technical paper, 2016-01-0701. 2016.

[20] Yamashitaa K, Saburib T, Wadab Y, Asaharaa M, Mogic T, Hayashia AK. Visualization of spontaneous ignition under controlled burst pressure. Int J Hydrogen Energy 2017;42:7755-60.

[21] Oran ES, Gamezo VN. Origins of the deflagration-to-detonation transition in gas-phase combustion. Combust Flame 2007;148:4-47.

[22] Molkov V, Verbecke F, Makarov D. LES of hydrogen-air deflagrations in a 78.5-m tunnel. Combust Sci Technol 2008;180:796-808.

[23] Verhelst V, Wallner T. Hydrogen-fueled internal combustion engines. Prog Energy Combust 2009;6:490-527.

[24] Li H, Karim GA. Knock in spark ignition hydrogen engines. Int J Hydrogen Energy 2004;29:859-65.

[25] Luo $Q$, Sun B. Inducing factors and frequency of combustion knock in hydrogen internal combustion engines. Int J Hydrogen Energy 2016;41:16296-305.

[26] Li J, Zhao ZW, Kazakov A, Dryer FL. An updated comprehensive kinetic model of hydrogen combustion. Int J Chem Kinet 2004;36:566-75.

[27] Maurya RK, Akhil N. Comparative study of the simulation ability of various recent hydrogen combustion mechanisms in $\mathrm{HCCl}$ engines using stochastic reactor model. Int J Hydrogen Energy 2017;42:11911-25.

[28] Olesky LM, Martz JB, Lavoie GA, Vavra J, Assanis DN, Babajimopoulos A. The effects of spark timing, unburned gas temperature, and negative valve overlap on the rates of stoichiometric spark assisted compression ignition combustion. Appl Energy 2013;105:407-17. 
[29] Yang H, Shuai S, Wang Z, Wang J. Fuel octane effects on gasoline multiple premixed compression ignition (MPCl) mode. Fuel 2013;103:373-9.

[30] Pan J, Shu G, Zhao P, Wei H, Chen Z. Interactions of flame propagation, auto-ignition and pressure wave during knocking combustion. Combust Flame 2016;164:319-28.

[31] Qi C, Dai P, Yu H, Chen Z. Different modes of reaction front propagation in $\mathrm{n}$-heptane/air mixture with concentration non-uniformity. Proc Combust Inst 2017;36:3633-41.

[32] Qi C, Chen Z. Effects of temperature perturbation on direct detonation initiation. Proc Combust Inst 2017;36:2743-51.

[33] Kee RJ, Grcar JF, Smooke MD, Miller JA. Sandia national laboratory report. SAND85-8240. 1985.

[34] Kee RJ, Rupley FM, Miller JA. Sandia national laboratory report. SAND89-8009B. 1989.

[35] Chen Z, Burke MP, Ju Y. Effects of Lewis number and ignition energy on the determination of laminar flame speed using propagating spherical flames. Proc Combust Inst 2009;32:1253-60.

[36] Chen Z. Effects of radiation and compression on propagating spherical flames of methane/air mixtures near the lean flammability limit. Combust Flame 2010;157:2267-76.

[37] Martz JB, Lavoie GA, Im HG, Middleton RJ, Babajimopoulos A, Assanis DN. The propagation of a laminar reaction front during end-gas auto-ignition. Combust Flame 2012;159:2077-86.

[38] Livengood JC, Wu PC. Correlation of autoi-gnition phenomena in internal combustion engines and rapid compression machines. Symp (Int) Combust 1955;5:347-56.

[39] Desantes JM, L opez JJ, Molina S, L opez-Pintor D. Validity of the Livengood \& Wu correlation and theoretical development of an alternative procedure to predict ignition delays under variable thermodynamic conditions. Energy Convers Manage 2015;105:836-47.

[40] Kapila AK, Schwendeman DW, Quirk JJ, Hawa T. Mechanisms of detonation formation due to a temperature gradient. Combust Theor Model 2002;6:553-94.

[41] Kagan L, Sivashinsky G. Autoignition due to hydraulic resistance and deflagration-to-detonation transition. Combust Flame 2008;154:186-90.

[42] Dziemi nska E, Koichi Hayashi A. Auto-ignition and DDT driven by shock wave e boundary layer interaction in oxyhydrogen mixture. Int J Hydrogen Energy 2013;38:4185-93.

[43] Liberman MA, Ivanov MF, Kiverin AD. Radiation heat transfer in particle-laden gaseous flame: flame acceleration and triggering detonation. Acta Astronaut 2015;115:82-93.

[44] Rudy W, Dziubanii K, Zbikowski M, Teodorczyk A. Experimental determination of critical conditions for hydrogen-air detonation propagation in partially confined geometry. Int J Hydrogen Energy 2017;42:7366-73. 